\title{
Tidal influence on high frequency harbor oscillations in a narrow
} entrance bay

\author{
S. Monserrat ${ }^{1,2}$, I. Fine ${ }^{3}$, A. Amores ${ }^{2}$, M. Marcos ${ }^{2}$ \\ ${ }^{1}$ Universitat de les Illes Balears, Departament de Física, Palma de Mallorca, Spain \\ ${ }^{2}$ Mediterranean Institute for Advanced Studies (IMEDEA), CSIC-UIB, Esporles, Spain. \\ ${ }^{3}$ Institute of Ocean Sciences (IOS), Sidney, British Columbia, Canada
}

Abstract: High frequency sea level oscillations at Wells Harbor (Maine, Northeastern US), with periods in the range of several tens of minutes, display a tidally modulated response. During low tides these sea level oscillations reach amplitudes of 10-20 cm, while during high tides they are significantly smaller. Wells Harbor is located in a low lying area with a tidal range of about $2 \mathrm{~m}$ and is connected to the open ocean through a narrow channel. Thus the extent and depth of the bay significantly vary over a tidal cycle. This changing geometry determines both the resonant periods and the amplification factor of the bay. Numerical results confirm the link between observed variability and these specific topographic features. Results imply that when exceptionally energetic long waves reach the Wells Harbor entrance (as in the case of a tsunami or meteotsunami) the expected response will be significantly stronger during low tide than during high tide. Although mean sea level would be lower in the former case, the currents inside the bay would be stronger and potentially more dangerous. This tidally modulated response could be extrapolated to other sites with similar topographic characteristics.

\section{1.- Introduction}

The topographic features of a bay or inlet determine how external energy is amplified under resonant conditions. The shape, bathymetry and configuration of the entrance to the bay define the characteristics of the major resonance modes, i.e. the seiche response, resonant frequencies and the inner basin quality factor $Q$ (Rabinovich, 2009).

The amplification factor $H$ for long waves arriving at the coast from the open sea may be approximately given by a single mode approach (Miles and Munk, 1961) 


$$
H^{2}=\left[\left(1-f^{2} / f_{0}^{2}\right)^{2}+Q^{-2} f^{2} / f_{0}^{2}\right]^{-1},
$$

33

where $f$ is the frequency of the long waves, $f_{0}$ is the fundamental resonant frequency of the bay (seiche), and $Q$ is the quality factor, $Q=2 \pi f_{0} / r$, with $r$ being the rate of frictional damping of the oscillations.

For a bay with a long narrow entrance channel, the fundamental frequency is determined by the equation

$$
f_{0}=\frac{1}{2 \pi} \sqrt{\frac{g}{l}} \frac{S}{A}
$$

where $S$ is the entrance cross-section, $A$ is the bay area, $l$ the channel length and $g$ the gravity acceleration (Miles and Munk, 1961; Raichlen, 1966). According to Eq. (2), the larger the bay area and the narrower the entrance for a given depth, the lower the fundamental frequency.

The quality factor $Q$, which is a linear measure of the energy damping in the system, depends on the size and shape of the bay. In the classical work by Miles and Munk (1961), the $Q$-factor is related to the radiation of the wave energy, but in a more general case, internal friction has also to be taken into account. The effective $Q$-factor of the system is then determined by both the frictional factor, $Q_{I}$, and the radiational factor, $Q_{R}$ (Garrett, 1975) where

$$
Q^{-1}=Q_{R}^{-1}+Q_{I}^{-1}
$$

For a narrow entrance, the radiation becomes negligible so that internal dissipation (friction) is the major effect controlling the quality factor (Raichlen, 1966).

In some locations the tidal range is large enough to significantly alter the bathymetric and geometric characteristics of the region, in particular when the surrounding coastal land is relatively flat. In such cases, the $Q$-factor and the expected amplification and damping of the incoming waves differ during low and high tide. According to Eq. (2), the resonant frequency for the bay may also differ within the tidal cycle: it becomes lower for high tide and higher for low tide. 
The tidal regime can have an additional effect on the $Q$-factor. Internal friction depends on the current speed and depth. So, in a tidally controlled estuary, where the major currents are related to the tides, highest speeds will occur in the bay channel during the peak ebb and flood tidal phases. Also, the tidal current amplifies when the bay area increases. So, the dissipation rate $r$ will increase when the bay area increases and the $Q$ factor will decrease even more strongly than in the case of a constant dissipation rate without considering the frictional effects.

As indicated by equation (1), when the $Q$-factor is much greater than unity, the amplification factor attains its maximum very close to $H^{2}=Q^{2}$ when $f=f_{0}$ (the approximation being very good for large values of $Q$ ) then decreases to unity as $f$ approaches 0 and goes to zero as $f$ goes to infinity (Fig. 1). When the Q-factor decreases the maximum moves towards the origin, being located at $f=0$ when $Q=1 / \sqrt{2}$. When $Q<1 / \sqrt{2}$ there is no maximum and the amplification factor is always smaller than 1.0. The wave amplitudes reduce at any non-zero frequency; i.e. $H$ monotonically decreases with increasing frequency. These features of the bay response to incoming waves could be better explained and estimated using an equivalent circuit analogy, as was done by Miles (1971) and Cummins (2013). However, all these features are limited to a single oscillator approximation; when frequency increases, the presence of higher modes essentially change the properties of the amplification function.

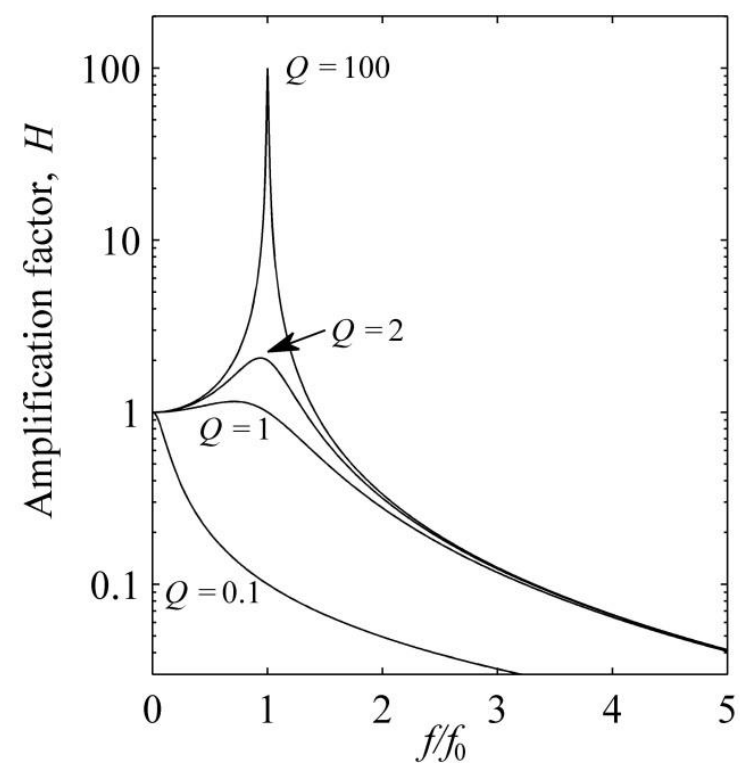

Figure 1: Amplification factors as a function of $f / f_{0}$ for different values of $Q$ 
81 It is important to quantify the resonant frequencies and the $Q$-factor for any bay or inlet

82 to evaluate the potential risk from energetic incoming open-ocean long waves, as in the 83 case of tsunamis or meteotsunamis. This can be easily done numerically by means of a 84 shallow water numerical model. The spectral ratio between oscillations inside and outside the bay enables us to estimate the resonant bay modes (seiches) and expected amplification ( $Q$-factor). (See, for example, Monserrat et al., 1998; Rabinovich et al., 1999; Liu et al., 2003).

Earlier studies have not addressed the effect of tidally modulated high frequency responses in semi-enclosed basins, likely because the effects were expected to be small. In this paper, we examine sea level observations at Wells Harbor (Maine, the northeastern coast of the United States) where the effect of the tidal range on the resonant properties of the bay is substantial. Wells Harbor is located in a low lying area with a tidal range of more than $2 \mathrm{~m}$. Here, the surface area of the bay is significantly influenced by changing mean sea level during a tidal cycle. The available observations and data analysis are described in Section 2. The observations are interpreted based on a numerical model described in Section 3. A comparison between the observations and numerical results is presented in Section 4. The implications of the observed phenomenon for the case of tsunami or meteotsunami, and extrapolation of the results to other regions, are also discussed.

\section{Observations}

In this study, we use tide gauge observations from two harbors with different geometries. Wells Harbor is located in in a lagoon in the Gulf of Maine (Fig. 2), in a flood plain with a tidal range of more than $2 \mathrm{~m}$ and connected to the open sea through a narrow channel (Fig. 3). As a result, the extent of this lagoon is significantly affected by changes in sea level during a typical daily tidal cycle. The nearby harbor at Fort Point (Fig. 2), whose geometry is not appreciably altered by the tidally varying sea level, was used for comparison. 


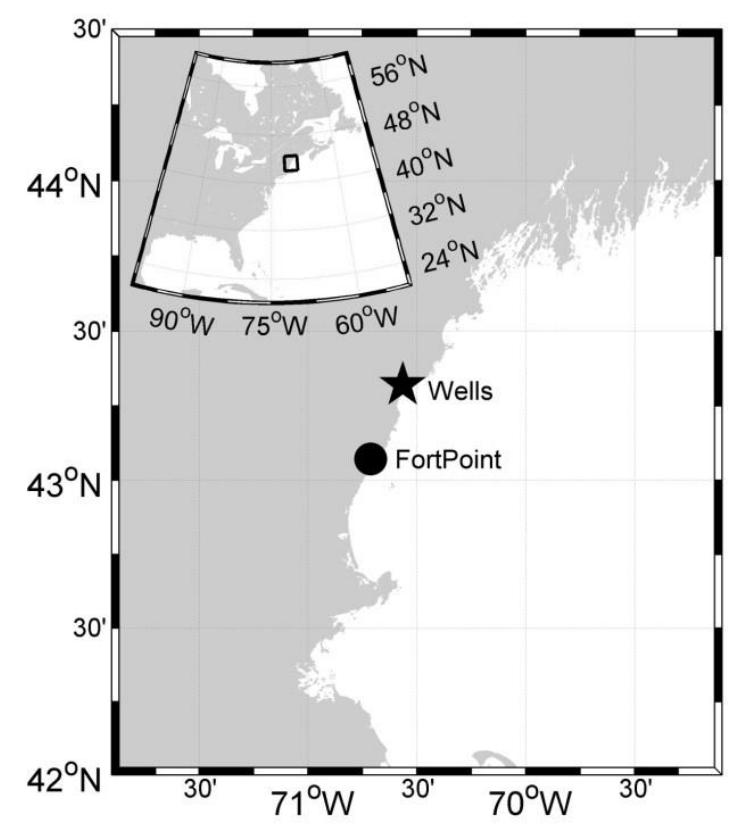

108

Figure 2: Location of the Wells Harbor and Fort Point tide gauges on the east coast of the United States.

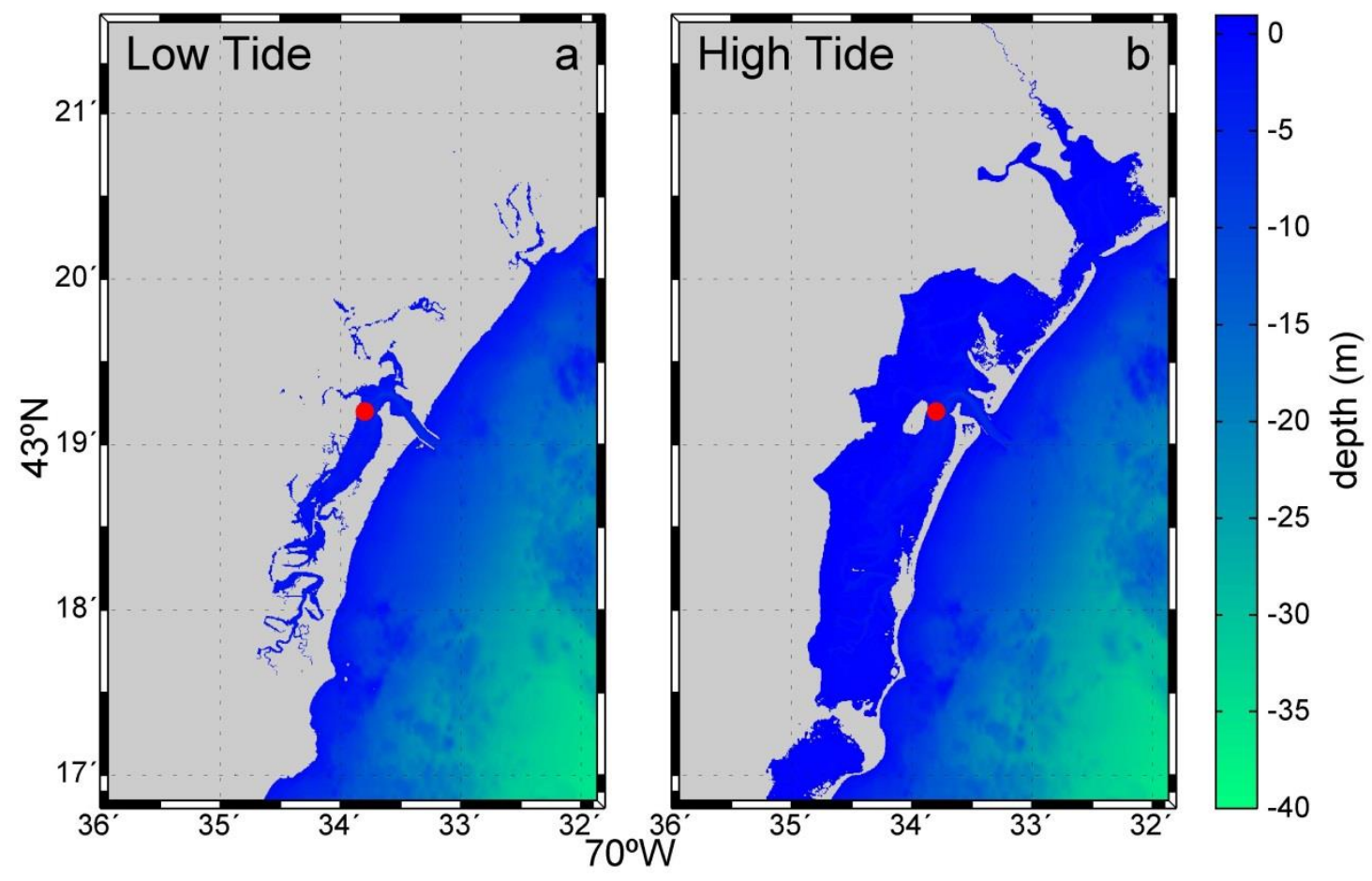

Figure 3: Bathymetry of the tidal basin containing the Wells Harbor tide gauge during low tide (a) and high tide (b). The maps have been computed by subtracting and adding $1 \mathrm{~m}$, respectively, to the mean harbor bathymetry. The location of the tide gauge is indicated by a red circle. 
117 Tide gauge sea-level observations in the Gulf of Maine were obtained from the NOAA 118 CO-OPS website http://opendap.co-ops.nos.noaa.gov. The sea level time series for 2008 119 used in this study for Fort Point and Wells have 6 min sampling intervals and $1 \mathrm{~mm}$ 120 precision. The raw data were visually checked for spikes, gaps and discontinuities. No 121 serious problem was detected. Some few missing points were linearly interpolated 122 before applying a further analysis.

123 The corrected sea level time series from Wells and Fort Point were de-tided and high124 pass filtered with 1-hour Kaiser-Bessel window. The observed oscillations with periods 125 of several tens of minutes and amplitudes of 10-20 cm (Fig. 4) correspond to harbor seiches. The amplified seiches that occurred in both harbors around midnight of 16

127 November 2008 are the result of a relatively small meteotsunami. Meteotsunamis have 128 already been reported in the region. In particular, the event of $28^{\text {th }}$ October 2008 has been extensively studied (Vilibić et al., 2013).

130
In addition to the strongest signal of the two sites being considered, the high frequency oscillations for Wells display a distinct tidal amplitude modulation, with energetic seiches during low tides and much weaker seiches during high tides (Fig. 4a). No such tidal modulation is observed at Fort Point in the neighboring harbor (Fig. 4b).

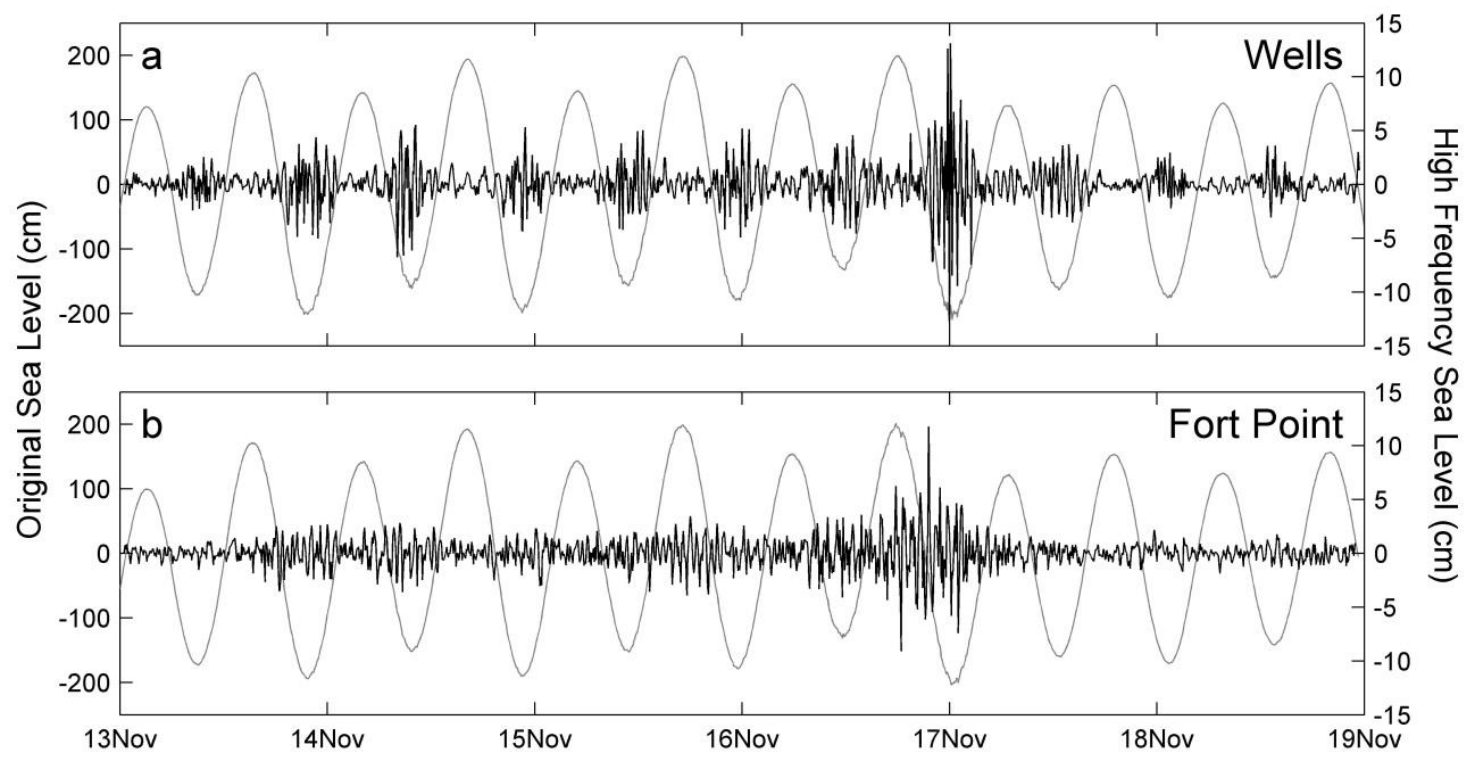


137 Figure 4: Original data time series (grey) and de-tided data (black) recorded at (a) Wells and (b)

138 Fort Point during November 2008. De-tided data have also been high-pass filtered with 1-hour window.

141 The meteotsunami of 16 November 2008 was observed at Fort Point during almost the 142 entire 12-hour tidal cycle but, in contrast, at Wells Harbor it is only apparent during the 143 low tide, becoming there even more energetic than at Fort Point. This response is 144 clearly associated with the pulse-like seiche behavior observed at Wells and related to 145 the tidal regime.

146 The frequency response of the de-tided time series at Wells was analyzed using a Morlet 147 wavelet (Fig. 5). Energy content in the range from $10 \mathrm{~min}$ to $2 \mathrm{~h}$ was computed for a 4.5 148 days period (November $13^{\text {th }}-18^{\text {th }}$ ). For comparison, we also show the tidal cycle in Fig. 149 5. Tidal amplitudes modulate not only the amplification response to the incoming 150 energy, which is higher during the low tide, but also the frequency of the seiche 151 oscillations. Therefore, when particularly large high frequency energy reaches the Wells 152 Harbor entrance (as in the case of the above mentioned meteotsunami), the expected response is significantly greater during low tide than during high tide. Although the mean sea level would be lower in the former case and the flooding risk reduced, the currents inside the bay would be much stronger and potentially more dangerous. 

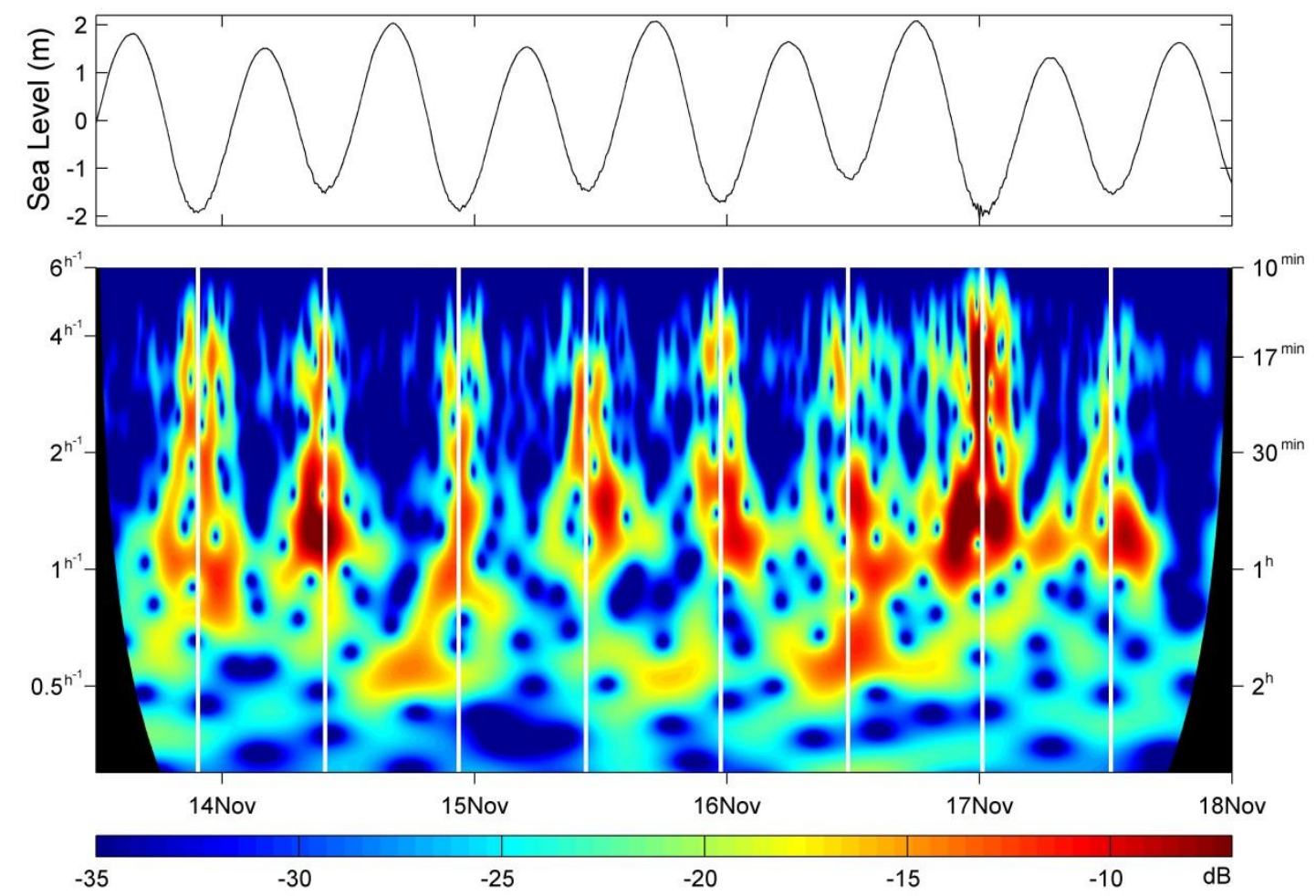

Figure 5: Wavelet analysis of sea level recorded at Wells over 4.5 days in November 2008.

Tides at this location are also shown and the times of low tide are indicated with vertical white lines in the wavelet plot.

\section{Numerical model}

A linear, shallow-water equation numerical model was used to compute the seiche response for two scenarios with bathymetries corresponding to a tide that varies by \pm 1 m (see Fig. 3). The bathymetry, with cell size of $1 / 3$ arc-second (about $10 \mathrm{~m}$ ), has been 172 obtained from the NOAA National Geophysical Data Center (Lim et al., 2009).

173 The model finite-difference formulation is similar to the one used in the TUNAMI N2 model (Imamura, 1996). The model was forced externally by specifying prescribed 
175 incident waves at the open boundaries. The incident waves had a "red noise" spectra, 176 similar to the observed background noise in the open ocean (Fine et al., 2009). The open 177 boundary radiation condition in the model has the form

178

$$
\frac{\partial \zeta}{\partial n}-\frac{1}{c} \frac{\partial \zeta}{\partial t}=y_{0}
$$

180

where $\zeta$ represents sea level elevation at time $t, c$ is the wave speed, $n$ is directed normal to the model boundary and $y_{0}$ is the forcing term at the entrance, which is computed using a stationary autoregressive (AR) model of the first order:

$$
y_{k}=a y_{k-1}+\varepsilon
$$

Here $y_{k}$ is the autoregression output which is then used as the open boundary condition, $a$ is the regression coefficient $(0<a<1)$, and $\varepsilon$ is a random "white noise" process. The normalized spectrum of $y_{k}$ (and consequently $\frac{\partial \zeta}{\partial t}$ ) has the form over time.

199 The spectrum (7) is a monotonic function of $f$, decreasing as $f^{-2}$ for frequencies less than $200 f_{a}$, where $f_{a}=-(2 \pi \Delta t)^{-1} \log (a)$ and with a higher rate (up to $f^{-4}$ ) for frequencies 201 higher than $f_{a}$. 
202

203

204

205

206

207

208

214

215

We have used $f_{a}=1 / 120 \mathrm{~s}^{-1}$ (i.e. the boundary frequency has been defined as $0.5 \mathrm{~min}$ ${ }^{1}$ ), thus, the incoming waves have a $f^{-2}$ dependence for the seiche frequency band.

The computational domain (Fig 3) has grid dimensions of 1190 rows $\times 1081$ columns and an $(x, y)$ grid size of $7.5 \mathrm{~m}$ by $10.3 \mathrm{~m}$ (or $1 / 3$ arc second in spherical coordinates). The time step used was $0.23 \mathrm{~s}$, and results were saved at prescribed grid points every 1 $\min$

To model the wave dissipation in the bay, we included a linearized Manning friction of the form

$$
r=2 g U n^{2} h^{-4 / 3}
$$

where $U(x, y)$ is a tidal current speed, $g$ is the acceleration of gravity, $n=0.03$ is the Manning coefficient and $h$ is the water depth. We have assumed that the tidal current dominates the flow and that the wave-induced velocity is parallel to the tidal velocity.

To estimate the tidal speed to be used in our simulations, we computed a low-frequency response during both low and high tide cases. Results are presented in Fig. 6. Due to the much larger water body during high tide, the simulated tidal currents are approximately five times stronger than at low tide for the same rate of sea level change.
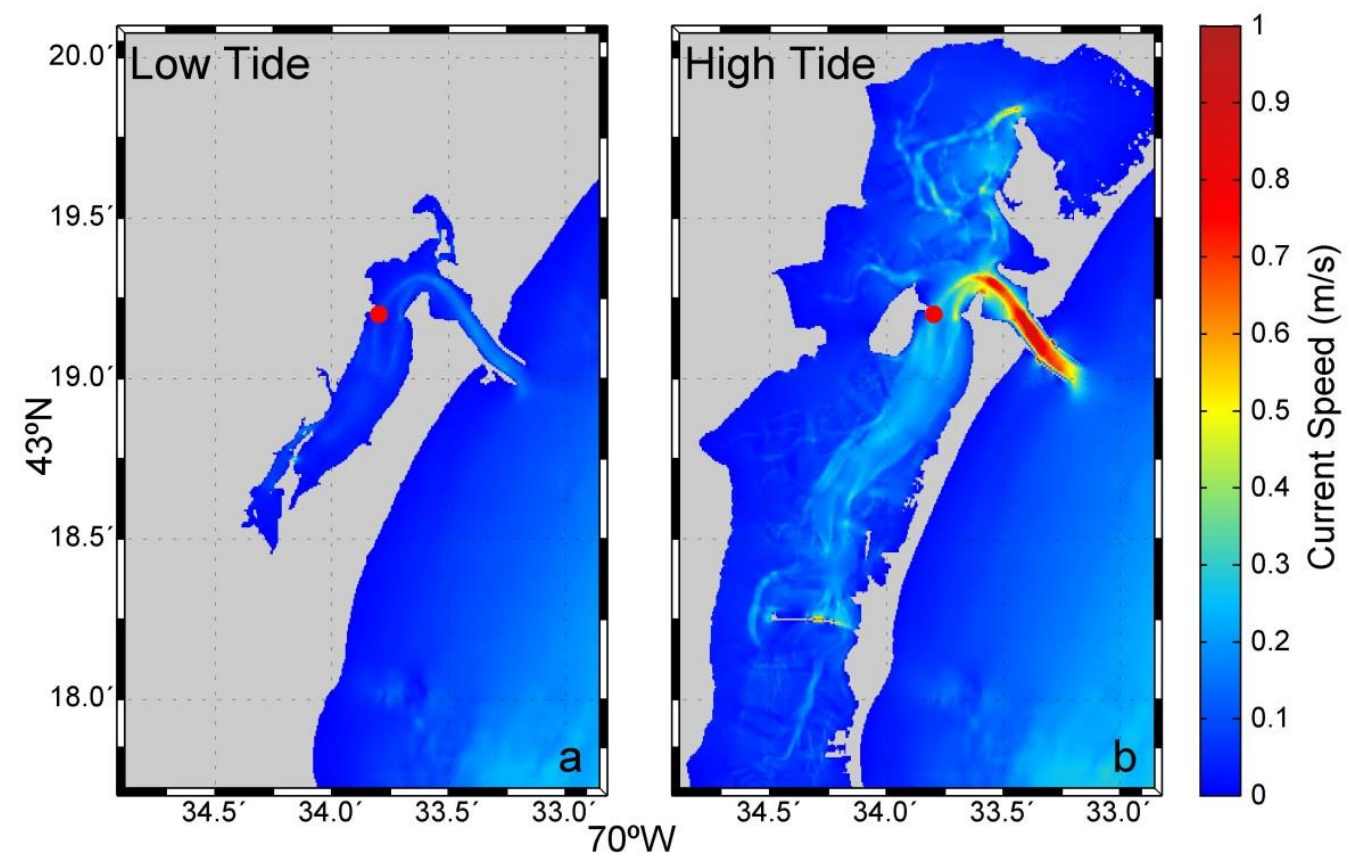

Figure 6: Numerically computed tidal current speeds during low and high tides. 
220 We simulated the bay response for $640 \mathrm{hrs}$ ( $\sim 27$ days) for two conditions corresponding 221 to low and high water. Results of the simulated waves at the grid points corresponding 222 to the tide gauge locations were stored for analysis.

223

224

\section{Discussion and conclusions}

225

226 The output of the numerical model and the tide gauge observations at Wells Harbor 227 have been compared in the frequency domain (Fig. 7). 


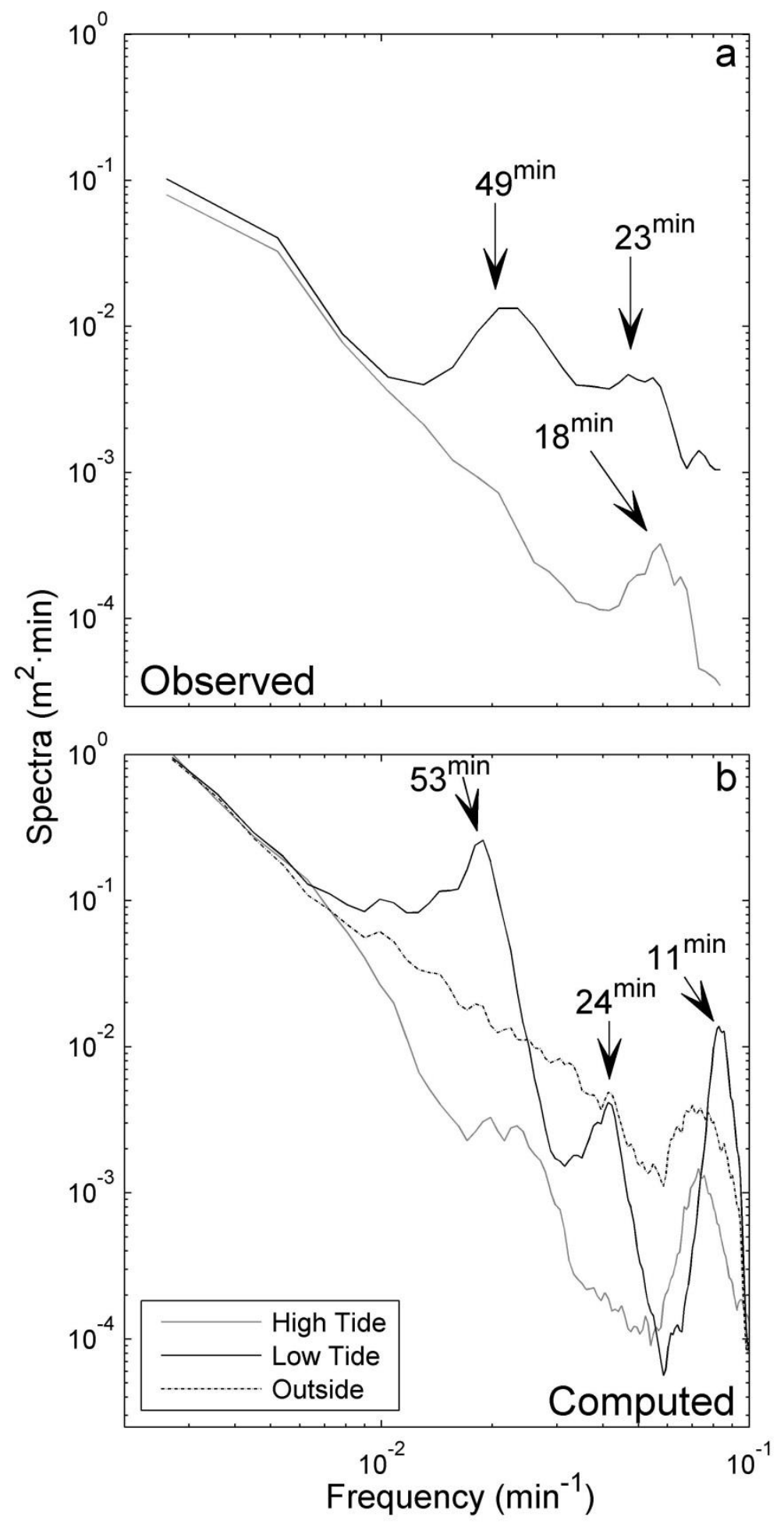

229 Figure 7: Sea level spectra during low and high tide at Wells Harbor using (a) observations and (b) numerical results. The spectrum for a site located outside Wells Harbor is shown in (b). Observational spectra have been computed by averaging of 50 individual spectra ( $\mathrm{DoF}=150$ ). Numerical spectra have $\mathrm{DoF}=148$. 
234 For the observations, spectra have been computed separately for low and high tides aiming to estimate the resonant characteristics for the two situations. To do so, 6.4-hour segments centered at high and low tides were selected. Since the reference sea level during low and high tide varies significantly due to the tropical-equatorial and springneap cycles, only those segments for which the reference sea level ranged between 1.1 $\mathrm{m}$ and $0.9 \mathrm{~m}$ above and below the yearly mean sea level have been selected. The spectra were computed with a Kaiser-Bessel window of $2^{6}=64$ points with 2 degrees of freedom (DoF) (Emery and Thomson, 2001). This was repeated a total of 50 times with segments randomly selected from those fulfilling the above mentioned criterion for one entire yearly record. Then, in order to decrease the confidence intervals, the corresponding spectra were averaged independently for high tide and low tide segments. The initial $\mathrm{DoF}=2 \mathrm{DoF}$ were subsequently increased to $\mathrm{DoF}=150$.

The limitation of the tidal cycle does not exist in the output of the numerical model, as this was run with a uniform sea level. We computed then the spectra with series of 38400 points ( 26.7 days) and used a half-overlapping Kaiser-Bessel window of $2^{10}=1024$ points. With these selected values we obtain $\operatorname{DoF}=148$, i.e. almost the same as for the observed spectra.

The observational spectra (Fig.7a) show a peak at about 18 min, detected in both low and high tide spectra, although it becomes more energetic and slightly displaced towards higher periods during low tide. The second peak, at about $49 \mathrm{~min}$, is only apparent and very energetic during low tide. The comparison between the two spectra demonstrates how the seiche response inside the harbor is changing through the tidal cycle. These changes are associated with the bathymetric changes in the harbor as the mean sea level varies.

The results for the simulated spectra for a site located outside the bay and at the tide gauge location are shown in Fig. 7b. During low tide, the spectrum significantly increases at the fundamental frequency (about $0.02 \mathrm{~min}^{-1}$, i.e period of $53 \mathrm{~min}$ ) as compared with the energy outside the bay. At higher frequencies, the spectrum inside the bay decreases faster than outside, thus the amplification factor becomes smaller than unity. At the first bay mode ( $24 \mathrm{~min})$, the energy of the low-tide seiche increases again but the amplification factor remains below 1.0. At high tide, the wave spectrum inside 
the bay is much lower than the spectrum outside the bay for all frequencies, indicating that the $Q$-factor is less than 1.0. The high frequency peak found around $11 \mathrm{~min}$ is above the Nyquist frequency for the observational spectra (12 min period) and could only be observed if the sampling rate for the observations was increased.

Comparing numerical simulations inside and outside the bay, the spectral amplification at the fundamental frequency during low tide can be estimated as $\sim 30$. Therefore, the $Q$ factor, following equation (1), should be of around 5-6, and accordingly, the linear damping rate would be approximately $0.02 \mathrm{~min}^{-1}$. The bottom friction is expected to be significantly higher at the entrance channel during high tide because the bay area is much larger and the cross-section of the channel remains almost unchanged. As a result, the estimated $Q$-factor for high tide would be as low as 0.4 .

It should be noticed that the wave spectra result from a topographic response combined with the incoming wave energy and, in this respect, the forcing of the model may differ from that for an actual event. To avoid this limitation, and remove the influence of the incoming wave energy in the comparison between observations and numerical results, we estimated the spectral ratios between low and high tide spectra (Fig. 8). Both "observational" and "computed" spectral ratios have quite similar shapes, but are not totally identical. The computed peak at $55 \mathrm{~min}$ is shifted towards higher frequencies in the observations (to a period of approximately $38.5 \mathrm{~min}$ ). However, the second peak at 23-24 min is clearly observed in both the computations and the observations. The actual values of the spectra are also similar, although those for the numerical values are slightly larger.

In general, the numerical results support our assumptions and demonstrate that the observed tide-modulated variability in seiche oscillations at Wells Harbor is definitively associated with changes in the basin geometry between low and high tides. The numerical model correctly reproduces the period of 23-min for the seiche oscillations affected by the bathymetric changes, although it slightly overestimates their actual amplification. The variation between the peaks and troughs in spectral ratios determined from the numerical results is also much more pronounced than in the observations. The observed differences are not surprising and could be attributed to the fact that the simulations were run with a constant reference sea level, whereas the actual tidal range 
is continuously varying during the 12-hour tidal cycle. It is expected then that spectra computed using observations will be smoother due to this averaging. A more sophisticated numerical model, including a "tidally modulated" reference sea level, would likely provide better agreement with the observations, but the effect we wanted to show becomes apparent enough even for a simple model with a constant bathymetry.

301

302

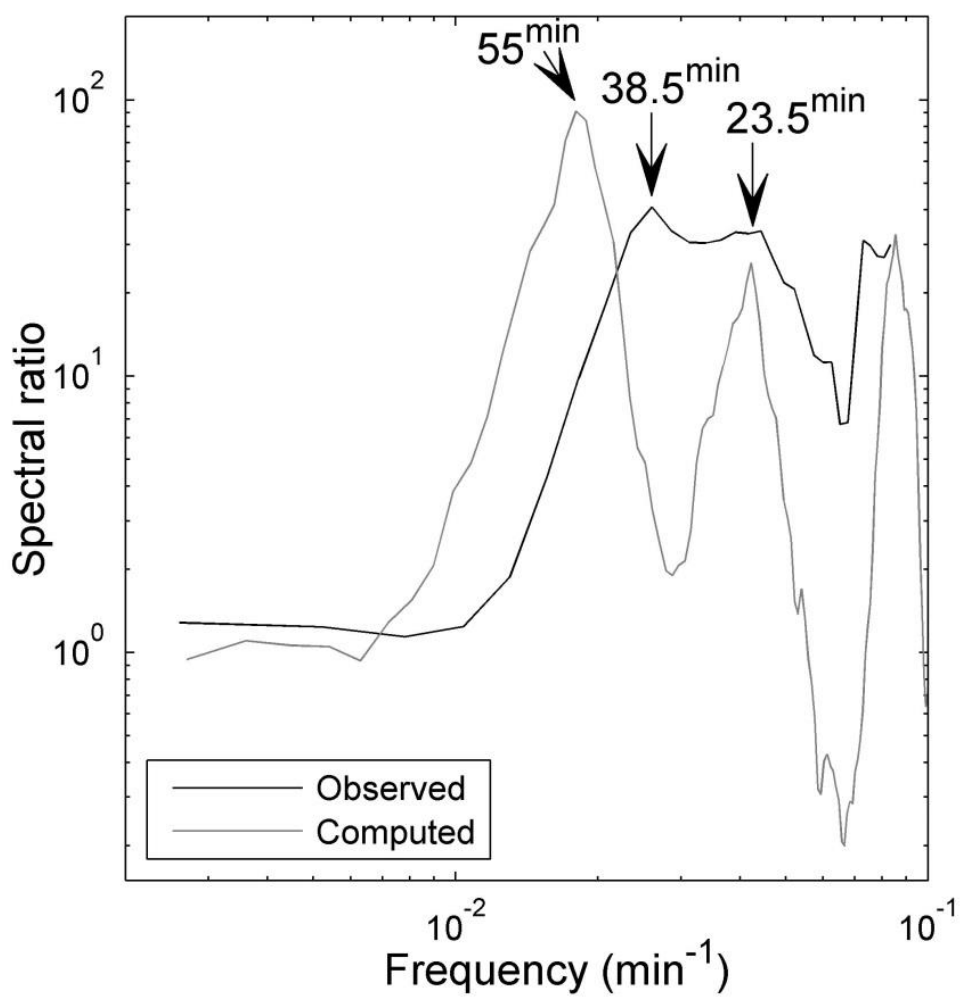

Figure 8: Spectral ratios between the spectra estimated for low and high tide series at Wells Harbor for both observations (black) and numerical computations (grey). 
Acknowledgments: We would like to thank the NOAA and the Gulf of Maine Research Institute, in particular John Jensenius and Linda Mangum, for their help in the acquisition of the data. Tide gauge data were obtained from the NOAA CO-OPS website at http://opendap.co-ops.nos.noaa.gov/axis/webservices. This work was partially performed within the NOAA/NWS project 'Towards a meteotsunami warning system along the U.S. coastline (TMEWS)', Award No. NA11NWS4670005. The work of A. Amores has been funded by a JAE-PreDoc grant from Consejo Superior de Investigaciones Científicas (CSIC) and co-funded by Programa Operativo FSE 20072013. M. Marcos acknowledges a "Ramon y Cajal" contract funded by the Spanish Ministry of Economy.

\section{References:}

Cummins, P.F. (2013) The extractable power from a split tidal channel: An equivalent circuit analysis, Renewable Energy, 50, 395-401.

Emery, W. J. and Thomson, R. E. (2001) Data Analysis Methods in Physical Oceanography, Second and revised edition, Elsevier, New York, 638 p.

Fine, I.V., Cherniawsky, J.Y., Rabinovich A.B. and Stephenson, F. (2009) Numerical modeling and observations of tsunami waves in Alberni Inlet and Barkley Sound, British Columbia. Pure Appl. Geophys., 165, 1019-2044.

Garrett, C. (1975) Tides in the gulfs. Deep Sea Res., 22, 23-35.

Imamura, F. (1996) Review of tsunami simulation with a finite difference method In: Long Wave Runup Models (eds. H. Yeh, P. Liu, and C. Synolakis), pp. 25-42 (World Scientific Publishing, Hackensack, N.J.).

Kulikov, E.A., Rabinovich, A.B., Spirin, A.I., Poole, S.L., and Soloviev, S.L. (1983) Measurement of tsunamis in the open ocean. Mar. Geodesy 6 (3-4), 311-329.

Lim, E., Taylor, L.A., Ealins, B.W., Carignan, K.S., Warnken, R.R. and Medley, P.R. (2009) Digital elevation model of Portland, Maine: Procedures, data sources and analysis. Service NOAA Technical Memorandum NESDIS NGDC-30. 
Liu, P. L.-F., Monserrat, S., Marcos, M. and Rabinovich, A. B. (2003) Coupling

341 between two inlets: observation and modeling. J. Geophys. Res., 108 (C3). doi:10.1029/2002JC001478.

Miles, J. (1971) Resonant response on harbours: an equivalent-circuit analysis. J. Fluid Mechanics, 40, 241-265.

Miles, J. and Munk, W. (1961) Harbor paradox. J. Waterways Harbor Division, ASCE, 87, 111-130.

347

Monserrat, S., Rabinovich, A. B., and Casas, B. (1998) On the reconstruction of the transfer function for atmospherically generated seiches. Geophys. Res. Lett., 25(12), 2197-2200.

350

Rabinovich, A.B. (2009) Seiches and harbor oscillations. In: Handbook of Coastal and Ocean Engineering (ed. YC Kim). World Scientific Publ., Singapore, pp 193-236.

Rabinovich, A. B., Monserrat, S., and Fine, I. V. (1999) Numerical modeling of extreme seiche oscillations in the region of the Balearic Islands. Oceanology, 39(1), 16-24.

Raichlen, F. (1966) Harbor resonance: Estuary and Coastline Hydrodynamics (ed. A.T. Ippen), McGraw Hill Book Comp., New York, 281-340.

Vilibić, I., Horvath, K., Strelec Mahović, N., Monserrat, S., Marcos, M., Amores, A. and Fine, I. (2013) Atmospheric processes responsible for generation of the 2008 Boothbay meteotsunami. Nat. Hazards. doi: 10.1007/s11069-013-0811-y 